\title{
Multidisciplinary care in pediatric oncology
}

This article was published in the following Dove Press journal:

Journal of Multidisciplinary Healthcare

30 May 2011

Number of times this article has been viewed

\author{
Mary Ann Cantrell' \\ Kathy Ruble ${ }^{2}$ \\ 'College of Nursing, Villanova \\ University, Villanova, PA, USA; \\ ${ }^{2}$ Department of Pediatric Oncology, \\ Johns Hopkins University, School of \\ Medicine, Baltimore, MD, USA
}

\begin{abstract}
This paper describes the significant advances in the treatment of childhood cancer and supportive care that have occurred over the last several decades and details how these advances have led to improved survival and quality of life (QOL) for children with cancer through a multidisciplinary approach to care. Advances in the basic sciences, general medicine, cooperative research protocols, and policy guidelines have influenced and guided the multidisciplinary approach in pediatric oncology care across the spectrum from diagnosis through long-term survival. Two case studies are provided to highlight the nature and scope of multidisciplinary care in pediatric oncology care.
\end{abstract}

Keywords: childhood cancer, chemotherapy, leukemia

\section{Introduction}

The trajectory of care for children with cancer includes diagnosis through treatment, including surveillance, rehabilitation, palliation, and end-of-life care. ${ }^{1}$ Progress in treatment of childhood cancer, once a nearly universally lethal disease, has led to an overall cure rate of nearly $80 \% .{ }^{1}$ Advances in the cure rates for childhood cancer have paralleled the progress in supportive and palliative care for this population. The advances seen throughout the field can be attributed to the multidisciplinary nature of care delivery and research. This paper describes some of the major advances in the treatment of childhood cancer and supportive care that have occurred over the last several decades and how these multidisciplinary advances have led to improved survival and QOL for children with cancer.

\section{The early years}

Pediatric oncology emerged as a subspecialty in the years following World War II when Farber observed the benefits of chemotherapy for acute childhood leukemia. ${ }^{3}$ Until then, general practitioners and pediatricians, in consultation with general surgeons, pathologists, and therapeutic radiologists, treated children with cancer, in which drug therapy consisted of single agents administered only to children with leukemia; children with solid tumors were not offered any systemic treatment. ${ }^{2}$ The science of pediatric oncology systemic therapy further advanced when Farber reported that remission was being achieved in children diagnosed not only with leukemia, but also with lymphoma and Wilms' tumor. ${ }^{3}$ Wilbur reported that chemotherapy could effectively reduce the size of solid tumors to enhance the effectiveness of other treatment modalities such as surgery and radiation. ${ }^{4}$ These early successes were
Correspondence: Mary Ann Cantrell College of Nursing, Villanova University, 342 Driscoll Hall, 800 East Lancaster Avenue, Villanova, PA 19085-1690, USA $\mathrm{Tel}+\mathrm{I} 6105196829$

Fax +16105197650

Email mary.ann.cantrell@villanova.edu 
the impetus for multidisciplinary therapy. Meadows pointed out that modern therapy for childhood cancer using multiagent and multimodal therapy began about $1970 . .^{5}$

\section{Disease-specific success stories}

Advances in the basic sciences, general medicine, cooperative research protocols, and policy guidelines have influenced and guided the multidisciplinary approach in pediatric oncology care. The treatment of leukemia is a good example of the multidisciplinary cooperation necessary to develop the successful regimens currently available to children diagnosed with leukemia. The use of antimetabolite chemotherapy to treat childhood leukemia emerged as an effective therapy in the 1960s, but the success was short lived and did little to improve the overall survival. ${ }^{6}$ The next advances in the treatment of childhood leukemia came with the addition of multi-agent therapies, but, again, long-term remissions were elusive due to central nervous system (CNS) involvement of the disease. ${ }^{7}$ The discipline of radiotherapy was incorporated to overcome this new obstacle and treatment/ prophylaxis of the CNS in children with leukemia was initiated. ${ }^{8}$ The addition of CNS radiation made dramatic improvements to the length of remission of leukemia, but as survival improved the intellectual disadvantages for survivors of this therapy emerged. As clinicians worked on designing therapies that could adequately treat the CNS with minimal impact on cognitive function, advances in the laboratory sciences became significant in the field. Immunologists were the first to identify prognostic markers in leukemia cells that would later dictate the therapies used and this led to the riskbased therapies used in today's regimens. ${ }^{9-12}$ Risk-based therapy allows treatment to be tailored so that children with the highest risk of poor outcomes receive sufficient therapy, while those at lower risk have therapy minimized. Leukemia remains a multidisciplinary success story, utilizing multi-agent, risk-based therapy and the prudent use of radiation to boast cure rates in excess of $80 \% .^{13}$

Multidisciplinary approaches have led to similar advances in most childhood cancers. The surgery discipline has been responsible for important advances in the treatment of many solid tumors. Osteosarcoma is the most common type of bone tumor seen in children and its management illustrates the important contributions the surgical discipline can make. ${ }^{14}$ Because osteosarcoma most frequently occurs in the extremities, it presents unique challenges for maintaining functionality without compromising cure. Limb salvage surgery is a crucial intervention necessary to insure optimal function for children and adolescents with this disease. Advances in surgical techniques, including state-of-the-art prostheses, allografts, and cadaver bone, have ensured complete removal of these tumors with good functional outcomes for survivors. ${ }^{15}$ Surgical advances in diagnostic and resection procedures for solid tumors have been important in the overall survival and QOL in childhood cancer. ${ }^{16}$

\section{Pediatric cancer centers}

Optimal outcomes in childhood cancer are not only due to therapeutic advances but also reflect the influence of published policy guidelines that have enhanced short-term and long-term outcomes for children and adolescents with cancer. Specifically, the published guidelines of the American Academy of Pediatrics for pediatric cancer centers was a major step forward for advancing the multidisciplinary care approach for children and adolescents with cancer. ${ }^{17,18}$ These guidelines, listed in Table 1, specify that a multidisciplinary team committed to providing an optimal level of the care for children and adolescents with cancer must exist and be functional in these centers. These centers house the collective expertise and networks of experienced researchers and a variety of health care professionals who recognize the

Table I American Academy guidelines for multidisciplinary teams in pediatrics for cancer centers ${ }^{\mathrm{a}}$

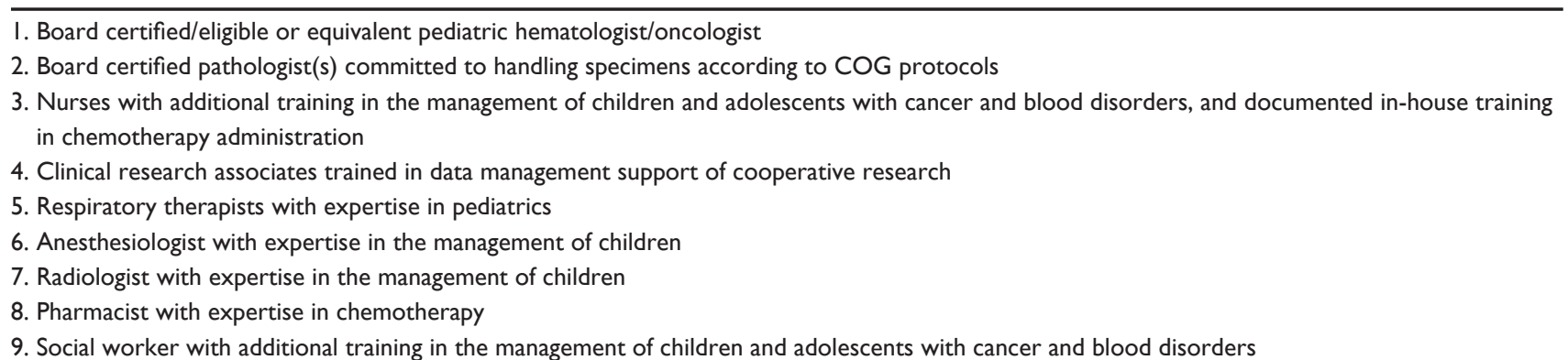

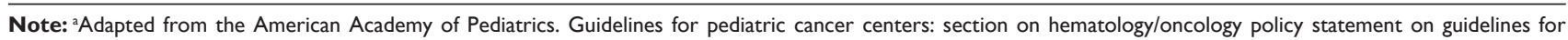
pediatric cancer centers. Pediatrics. 2004;| |3(6):|833-1835.

Abbreviation: COG, Children's Oncology Group. 
significance of randomized clinical trials as the most effective method for identifying more successful treatment strategies and who have the resources to evaluate new treatment modalities. ${ }^{18}$ These multidisciplinary teams offer a unique and the most effective approach to the early detection, accurate diagnosis, and appropriate treatment for various childhood cancer diagnoses.

Pediatric cancer centers with comprehensive multidisciplinary teams are often limited to developed countries and far fewer resources are available in underdeveloped areas of the world. It is expected that only $25 \%$ of children diagnosed with cancer in low- to middle-income countries will survive. ${ }^{19}$ The creation of a multidisciplinary pediatric oncology unit, utilizing protocol-based therapy and local support has been shown to nearly double (from $32 \%$ to $63 \%$ ) the 5 -year eventfree survival in a region with otherwise limited resources. ${ }^{20}$ In developed countries there is some controversy over the equality of the benefit of multidisciplinary care. For example, nearly every child treated for cancer in the USA can expect to receive treatment at a pediatric cancer center, yet racial differences in survival can still be observed. ${ }^{21}$ In these circumstances, multidisciplinary care alone may not be enough to overcome the environmental, social, and biological differences among children with cancer.

The medical care and management of childhood cancer does not reside solely in the specialty disciplines at pediatric cancer centers. Primary care physicians in the community have an important role throughout the trajectory of childhood cancer care. The pediatrician is typically the first to evaluate symptoms associated with cancer; this is not an easy task, as many of the initial symptoms mimic common childhood illnesses. Fever, abdominal mass, lymphadenopathy, headache, bone pain, and abnormal blood counts are associated with newly diagnosed childhood cancer; the expertise of a skilled practitioner is required to differentiate these symptoms from the numerous non-malignant conditions that have similar presentations. ${ }^{22}$ The pediatrician's role does not end at diagnosis but continues throughout the treatment period to include the management of infection and treatment of side effects, as well as after-therapy care when monitoring for complications and ensuring appropriate growth and development are vital components of care. ${ }^{22,23}$

The contributions of pediatric oncology nurses in the multidisciplinary care of children with cancer are well recognized and valued. Klein described an interdisciplinary team should consist of practitioners from different professions who share a common patient population and common patient care goals and have responsibility for complementary tasks. ${ }^{24}$ Specific outcome-based advances in pediatric oncology nursing practice have augmented the prescribed treatments determined through clinical trials to address some of the common side effects and complications of agents administered in these protocols. One such example is a comprehensive, multi-focused project embarked upon by the Oncology Division of the Children's Hospital of Philadelphia to reduce chemotherapy errors. ${ }^{25}$ A specific venture within this multifocal project was the implementation of the "Rapid Hydration Protocol." This interdisciplinary research project was developed and tested by the pediatric oncology nursing staff. The outcomes of this evidence-based practice project were threefold: (1) decreased the time needed for hydration and the number of nurses involved in the institution of a chemotherapy protocol; (2) contribution made to having chemotherapy begin earlier in the day; and (3) systems in ordering chemotherapy protocols were improved and decreased, which reduced handoffs. ${ }^{25}$

The development and implementation of a formal structure for nursing research within the Children's Oncology Group (COG) structure was launched to enable more direct contributions of the nursing discipline to the scientific mission of this cooperative group. ${ }^{26}$ The strategic plan for this project was launched at the first State of the Science Summit for Pediatric Oncology Nursing Research on the campus of the National Institutes of Health in 2000. Four areas of research were identified: (1) the neurocognitive consequences for the treatment team, (2) fatigue and related symptoms, (3) the coping efforts of patients/families/team and (4) self-care. ${ }^{27}$

One published study that addressed fatigue and related symptoms examined the effects of dexamethasone on sleep and fatigue in which the lead investigator was a pediatric oncology nurse-researcher. ${ }^{28}$ This investigation involved 100 pediatric patients with low- or standard-risk acute lymphoblastic leukemia (ALL) enrolled in one of three COG protocols at three different institutions. It was reported that dexamethasone treatment during continuation therapy for childhood ALL significantly and adversely altered sleep and fatigue, confirming that sleep and fatigue are behavioral responses to dexamethasone. ${ }^{28}$ The next step, based on these findings, is to examine the relationship between these behavioral indicators and the biologic indicators of individual responsiveness to dexamethasone to identify pediatric patients with ALL who will be the most sensitive to dexamethasone treatment, thereby allowing clinicians to design optimal dosing schedules for individual patients. ${ }^{28}$ 
Despite these growing efforts, very few outcome-based studies to direct psychosocial care interventions for children and their families currently in treatment for cancer have been conducted. This is of great concern because pediatric oncology nurses provide significant psychosocial care to these patients. In a review of the trajectory of pediatric cancer research, Reaman noted that psychosocial and biobehavioral research on outcomes is missing in cooperative group studies. ${ }^{29}$ A major contributing factor to the lack of psychosocial and biobehavioral research using the cooperative group mechanism is limited restricted resources. Studies investigating psychosocial outcomes and biobehavioral interventions must compete with both clinical trials focused on survival improvements for participant enrollment for resources and group support for investigator time. ${ }^{29}$

In his review of the history of pediatric cancer research, Reaman noted that, to date, the bulk of therapeutic research in pediatric cancer accomplished with controlled clinical trials has focused on survival. ${ }^{29}$ Outcomes-based nursing research and subsequent nursing care for childhood cancer survivors have reflected these research efforts. Ruccione described the rich heritage in cancer survivorship research and outcomes-based clinical care, which was pioneered by the discipline of pediatric oncology and has had pediatric oncology nurses at the forefront of survivorship clinical care, research, and education for more than 30 years. ${ }^{30}$ Ruccione chronicled these accomplishments and highlighted milestones in outcomes-based clinical nursing care for survivors of childhood cancer by decades. Specifically, in the 1970s and 1980s, the establishment and growth of specialized long-term follow-up programs and the active involvement of nurses as members of cooperative group study committees in designing risk-adjusted therapy protocols aimed at minimizing late effects without decreasing survival were achieved. ${ }^{30}$ In the 1990 s and into the 2000 s, publications by pediatric oncology nurses about the late effects and risk-based follow-up care models of care for health care professionals and for the pediatric cancer survivor population were produced and disseminated. ${ }^{30}$ In the 2000s, a collaborative effort between the COG Nursing Discipline and the Late Effects Committee produced riskbased follow-up guidelines, a directory of services, and a resource guide, as well as launching several research protocols. $^{30}$

\section{QOL and multidisciplinary care}

Health-related quality of life (HRQOL) is important for assessing the successful treatment of childhood cancer.
HRQOL is a multidimensional construct that includes the impact of childhood cancer on the physical and psychosocial aspects of daily life for patients and families. Important components of HRQOL that have been identified during treatment include pain, nausea/vomiting, anxiety, concerns about communication, changes in body appearance, and cognitive dysfunction. ${ }^{31}$ While no studies were identified that specifically evaluated the impact of multidisciplinary care on HRQOL in this population, it is clear that addressing these issues requires expertise, including symptom management and psychosocial support from medical and allied health professionals. Models for survivorship care have embraced the multidisciplinary model and its potential impact on HRQOL. ${ }^{32}$ Many of the morbidities associated with childhood cancer survivorship have been shown to impact HRQOL, and can include organ damage, cognitive impairments, and psychosocial dysfunction. ${ }^{33-35}$ A multidisciplinary approach to the complex health care needs of childhood cancer survivors has been proposed to be an efficient way to deliver care that is beneficial to patients, providers, and institutions. $^{22}$

\section{Cooperative clinical trial groups}

Perhaps no other phenomenon has influenced the multidisciplinary approach to pediatric oncology care as much as the development and continued existence of pediatric oncology clinical group trials. Cooperative group research is essential to success in areas such as childhood cancer, where relatively small incidence rates require multi-institutional collaboration. Reaman noted that the pediatric clinical trials groups have a highly systematic and organized approach to the investigation of treatment strategies for children and adolescents with cancer through hypothesis-driven clinical trials using a multi-center approach. ${ }^{32}$ Prior to the establishment of cooperative groups, only Phase I or Phase II clinical trials using single agent therapy existed. Multi-agent therapies were begun in Phase III clinical trials through cooperative research groups and have accelerated the advances in treatment success. ${ }^{2}$

The first cooperative group in pediatric oncology was the Children's Cancer Group (CCG). The CCG was founded in 1955 with federal government funding by the National Cancer Institute (NCI). The CCG was a multi-institutional cancer research organization composed of 112 member institutions in the USA, Canada, and Australia. The overall goals of the CCG were to improve survival and QOL for cancer patients and conduct therapeutic trials in multidisciplinary fields. The CCG served as a base from which to 
conduct childhood cancer research through a team approach, including the diagnosis, treatment, and supportive care for children with cancer. ${ }^{37}$

Meadows identified landmarks in pediatric oncology by decade from the 1970s through the 1990s and linked these milestones to the efforts and outcomes of cooperative clinical trial groups. ${ }^{5}$ Landmarks in the 1970 s included recognition that cure was possible, proliferation of randomized clinical trials, and use of effective multimodality therapy. ${ }^{5}$ Treatment and advances in the 1980s included tailoring therapy to risk factors, defining late effects, using lower radiation doses, and substituting effective drugs for radiation. ${ }^{5}$ The $1990 \mathrm{~s}$ sentinel events in pediatric oncology, identified by Meadows, were the understanding of the relationship of dose to late effects, distinguishing research from clinical care, and initiating efforts to track and educate survivors. Additional contributions from these early cooperative research groups were the development of criteria for evaluation of response to therapy and the emergence of the biostatistician as an integral member of the research team.

Following the establishment of CCG, three other pediatric oncology clinical trials groups were formed: the Pediatric Oncology Group, the National Wilms' Tumor Study Groups, and the International Rhabdomyosarcoma Study Group. In 2000, the Children's Oncology Group (COG) was established through the merger of these four cooperative clinical trials groups with the goal of conserving resources and increasing accrual rates so that important clinical questions could be more quickly answered. It is estimated that a member institution of COG treats $90 \%$ of children diagnosed with cancer in the USA. ${ }^{38}$ To further validate the effectiveness of a multidisciplinary care in pediatric oncology, the COG has established guidelines for comprehensive pediatric hematology/oncology programs. The guidelines for required on-site personnel and on-site services are listed in Table 2.

\section{Supportive and palliative care}

Supportive and palliative care plays a crucial role in the successful management of childhood cancer. Infection, nausea/vomiting, and pain are three areas in which supportive care has experienced significant advances from a multidisciplinary approach. The morbidity and mortality associated with infection during treatment of childhood cancer remain serious obstacles. Nearly $3 \%$ of children with fever and neutropenia during cancer therapy are expected to die of sepsis, although progress in infection control has dramatically decreased morbidity and mortality during childhood cancer treatment. ${ }^{39}$ Bacterial, viral, and fungal infections pose risks to the child with cancer and the development of new and more effective pharmacologic options, especially anti-fungal and anti-viral agents, have dramatically decreased the morbidity and mortality associated with these infections. ${ }^{40,41}$ Most recently the identification of

Table 2 Required on-site services for the COG membership ${ }^{a}$

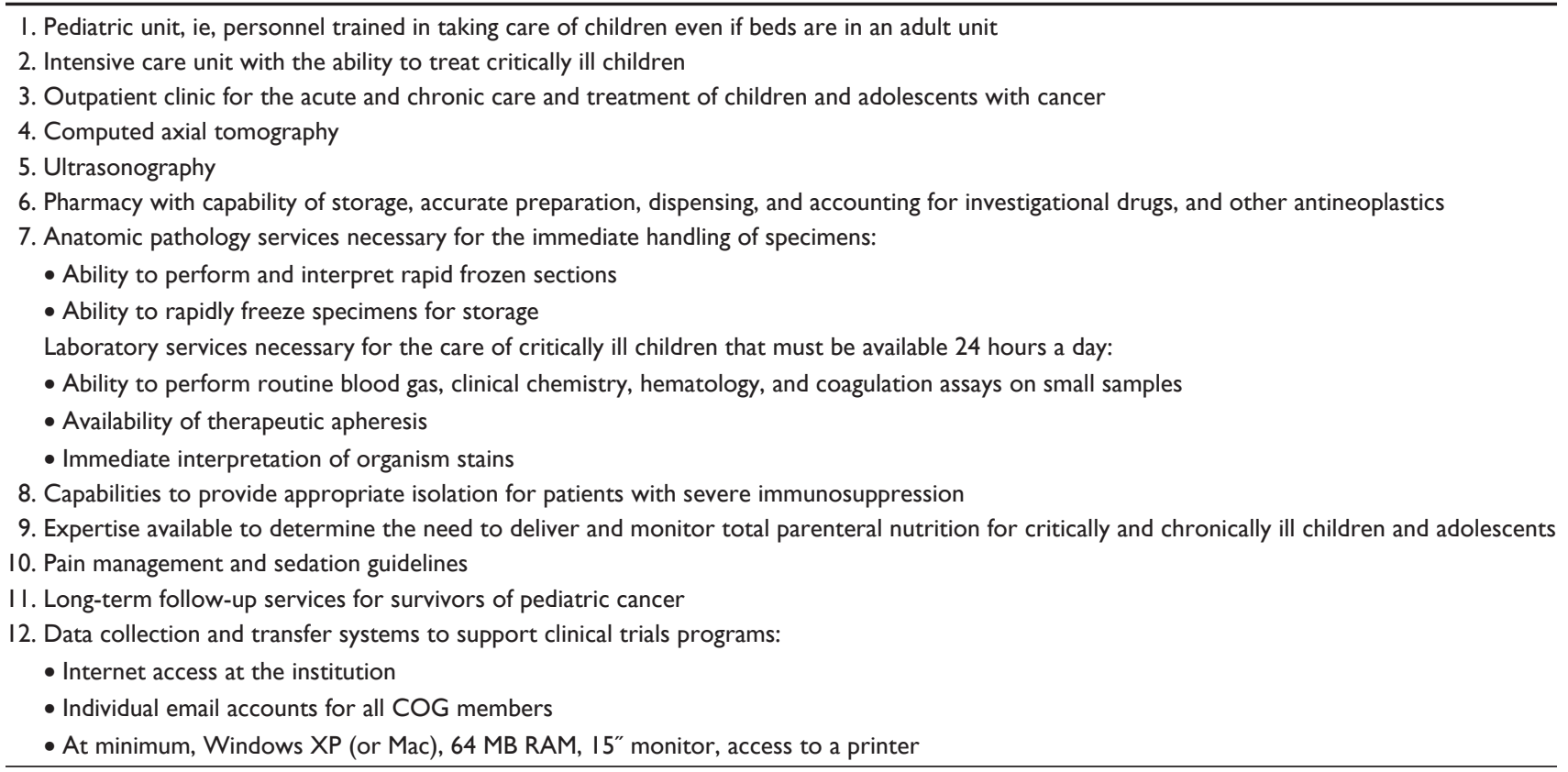

Note: aAdapted from Children's Oncology Group. ${ }^{64}$

Abbreviation: COG, Children's Oncology Group. 
children at highest risk for sepsis has been an important development and has led to early and aggressive treatment for the most vulnerable oncology patients. ${ }^{42,43}$

Other important advances in the prevention and treatment of infection in the childhood cancer population can be attributed to nursing care and research. Nurses are primarily responsible for the progress in patient/family education that ensures that techniques for preventing infection are followed and that early signs of infection are managed appropriately inside and outside the hospital setting. ${ }^{44}$ In addition, nurse researchers have contributed to the progress made in monitoring immunosuppressed patients and procedures to reduce the bloodstream infections often associated with central venous catheters. ${ }^{44-46}$

Multidisciplinary advances have been made in other supportive care areas that have led to dramatic improvement in QOL for children with cancer. Control of vomiting is one such area of significant achievement. Once only a handful of moderately effective agents were available to treat this side effect of cancer treatment in children. However, in the late 1980s a new class of antiemetic, the $5-\mathrm{HT}_{3}$ antagonists, began to be studied in children receiving chemotherapy and led to complete or major control of vomiting in $87 \%$ of the children studied. ${ }^{47}$ Since these early studies, physicians and nurses have worked side by side to better define, measure, and control not only vomiting, but also the equally unpleasant side effect, nausea. ${ }^{48,49}$ These efforts have led to valid measurement tools and multi-agent antiemetic regimens that minimize the impact of nausea and vomiting on QOL during childhood cancer treatment. ${ }^{50-52}$

Pain control is yet another critical component of supportive care in childhood cancer. Children may experience pain across the continuum of cancer care. It may be the initial symptom of their disease, a side effect of treatment, associated with procedures, or a symptom in the end-of-life phase for those who are not cured of their disease. Once again, a multidisciplinary approach is necessary to tackle this complex and serious threat to QOL. In the mid 1990s the World Health Organization (WHO) consulted experts in anesthesiology, neurology, nursing, oncology, pediatrics, pastoral care, and psychology to develop the Cancer Pain Relief and Palliative Care in Children consensus guidelines. ${ }^{53}$ These guidelines provide a ladder approach to pain management using progressively stronger analgesic agents while recognizing the importance of nondrug therapies. Since this time, the WHO analgesic ladder has been used to study and reduce the pain associated with childhood cancer throughout the world. ${ }^{54,55}$

\section{Complementary and alternative medicine}

The use of complementary and alternative medicine (CAM) in conjunction with standard medical treatment is an emerging integrative approach in the care of oncology patients. ${ }^{56}$ This integrative approach seeks to improve the supportive care available to patients while also determining through scientific clinical trials which adjuvant CAM therapies are medically sound, effective, and compatible with standard chemotherapy and radiation. ${ }^{57}$ Among pediatric oncology patients, CAM mind-body interventions are most often used as supportive care therapies to relieve symptoms, reduce side effects of treatment, and cope with the emotional aspect of living with a life-threatening disease. ${ }^{58}$ The interventions include hypnosis for reducing pain, anxiety, nausea, or vomiting, ${ }^{58,59}$ and music therapy and massage for improving mood states, anxiety, and symptom distress. ${ }^{57}$

With the increased use of CAM among pediatric oncology patients, investigations about its prevalence, safety, and effectiveness have emerged. Bishop and colleagues conducted a systematic review of published studies from 1975 to 2005 , which included 28 studies with survey data to summarize the current evidence on the prevalence of CAM among children with cancer. ${ }^{60}$ The total sample size across all 28 studies included 3526 pediatric oncology patients in whom the prevalence of CAM usage ranged from $6 \%$ to $91 \%$. In contrast to what was reported by Post-White, ${ }^{57}$ Bishop and colleagues reported that herbal remedies were the most often reported CAM modality used for these children, followed by diets/nutrition and faith healing. ${ }^{60}$

The COG has made a major commitment to CAM research in childhood and adolescent cancer, beginning with studies of CAM in the area of supportive care. ${ }^{61}$ The first randomized, double-blind, placebo-controlled clinical trial initiated in 2004 by COG assessed the efficacy of Traumeel-S ${ }^{\circledR}$ (Heel, Inc, Albuquerque, NM), a homeopathic remedy made from plant extracts for the prevention and treatment of mucositis in children undergoing stem cell transplantation. ${ }^{61}$ Post-White, Hawks, O'Mara, and Ott cited that the future directions in moving the CAM research agenda forward for children with cancer include: determining the safety, efficacy, and outcomes of individual CAM interventions, establishing safe dosages for children, and determining potential mechanisms of action and interactions with medical treatment. ${ }^{62}$ 


\section{Survivorship}

The multidisciplinary approach in pediatric oncology extends to the care of childhood cancer survivors. The Institute of Medicine's (IOM) report entitled Childhood Cancer Survivorship: Improving the Care and Quality of Life recognizes that the treatment of childhood cancer is one of oncology's great success stories. ${ }^{1}$ The IOM report also highlights that the unintended consequences of this success are not widely recognized. Intensity and complexity of treatment protocols and increasing survival rates among children and adolescents diagnosed with cancer has led to an increased awareness of the need to monitor and treat the long-term complications of these therapies. It is estimated that two-thirds of survivors will have at least one long-term complication and one-fourth will have a severe or life-threatening complication. ${ }^{1}$ These long-term complications have the potential to negatively affect not only the health status, but also the QOL for survivors. Zebrack and Zeltzer recognized that survivorship data provide little information about the QOL expected, enjoyed, or endured by these individuals. ${ }^{63}$ The IOM report identified health status and QOL as essential treatment outcomes for this population, who require a multidisciplinary approach to care. The Association of Cancer Online Resources now lists 53 comprehensive follow-up programs for childhood cancer survivors. The Association of Cancer Online Resources criteria required to be considered a comprehensive follow-up program include the requirements that the program: have a dedicated time and place for the survivor clinic to take place; meets at least twice a month; is staffed by a doctor with experience in the late effects after treatment for childhood cancer; has a nurse coordinator; and offers state-of-the-art screening for individuals' risks of late effects, referrals to appropriate specialists, and wellness education. ${ }^{64}$ In addition, CureSearch (associated with COG), provides information on 146 participating institutions that provide services for childhood cancer survivors and membership in COG requires that services be available for survivors. ${ }^{65}$

Multidisciplinary care for childhood cancer survivors has also been supported by research findings from the Childhood Cancer Survivors Study (CCSG). Established in 1993, the CCSG is a National Cancer Institute-funded cohort for the study of the long-term morbidity and mortality associated with the treatment of childhood cancer. Data are collected from a 26-member consortium of clinical pediatric centers in the USA and Canada; the database includes more than 14000 survivors and 3700 sibling controls. Publications resulting from CCSG data have addressed clinical practice issues, ranging from health care utilization, health behaviors, health status, chronic health conditions, psychosocial and QOL factors, second malignancies, endocrine and reproductive outcomes, to late mortality. ${ }^{66}$

The Long-term Follow-up Guidelines published by COG are yet another example of multidisciplinary progress for childhood cancer survivors. ${ }^{67}$ The guidelines, first developed in 2002, provide information on 136 therapeutic exposures, and 101 screening recommendations. They were developed by 18 multidisciplinary task forces that included physicians, physical therapists, nurses, audiologists, patient advocates, epidemiologists, social workers, and psychologists. Recommendations are based on scientific evidence and the collective experience of experts in the area. They are regularly updated to reflect new evidence in the literature. The goals of these guidelines are to promote healthy lifestyles, suggest appropriate ongoing monitoring, facilitate early detection of complications, and allow for timely interventions.

\section{Case studies}

This first case study describes the initial experience of a previously healthy 4-year-old boy named Andrew, who has been referred to a pediatric cancer center for a consult to evaluate the diagnosis of childhood leukemia by his primary care pediatrician. Andrew is directly admitted to the pediatric oncology unit of a pediatric cancer center. Within several hours of being admitted, he is scheduled to have a bone marrow aspirate and biopsy, as well as a spinal tap to confirm the diagnosis. This initial treatment intervention involves a multidisciplinary team approach involving a member of the medical oncology team, a pediatric oncology nurse, and a child life therapist. The oncologist and the pediatric primary nurse provide the family with the necessary education about the procedure. Likewise, the nurse and the child life therapist use therapeutic play procedures before, during, and after the procedure to educate Andrew and address his fears and concerns. The specimens obtained from the procedure are then taken to an on-site lab dedicated to the care of pediatric oncology patients and examined by laboratory technicians specially trained in the examination of specimens for childhood cancer. Following the confirmation of the diagnosis of childhood leukemia, a parent meeting is scheduled to share with Andrew's parents the diagnosis and plan of care. This meeting involves Andrew's primary oncologist, his primary nurse, and a social worker. While this meeting is occurring, a pharmacist with specialized training in chemotherapy agents is dispensing the protocol's chemotherapy. In addition, 
the pediatric surgery department has been consulted for placement of a central venous catheter.

Andrew's treatment is a risk-based therapy protocol based on gender, age, initial presenting white blood cell count (WBC), and the morphological, immunological, and genetic subtype classifications of his disease. Based on Andrew's age of 4 years, his initial WBC count of $22000 / \mathrm{mm}^{3}$ and favorable marrow cytogenetic finding of hyperdiploidy $>50 ; \mathrm{t}$ $(12 ; 21)$ he is considered to be standard-risk precursor B-cell ALL. Andrew is placed on a COG protocol that stratifies treatment for children with leukemia based on risk at diagnosis in which he will not receive anthracycline during his initial 28-day treatment plan. This initial treatment plan includes the following drugs and schedule: cytarabine intrathecally (IT) on Day 1, vincristine IV on Days 1, 8, 15, and 22, dexamethasone - IV or orally - twice daily on Days 1 through 28, pegaspargase intramuscularly (IM) on Day 4, 5 , or 6, and methotrexate IT on Days 8 and 29.

In the first week of this initial plan, Andrew also receives supportive care therapy to stabilize his physiological processes with a focus on his immune and hematological functioning. His therapy includes intravenous fluids, blood product transfusions, and vigil assessments of his vital signs and complete blood cell count (CBC) results. In addition to these supportive treatment measures, Andrew and his family receive psychosocial supportive care and are introduced to the following members of the treatment team and their specific roles.

- Pediatric oncology nurses. These specialized professional nurses provide care, support, and education to children and their families who have a variety of cancer diagnoses. Some institutions assign patients and their families a primary nurse who will serve as a consistent presence throughout the duration of their treatment. Andrew and his family also may have care needs that require the expertise of the nursing staff from the pediatric intensive care unit, the pheresis center, physical rehabilitation unit, and the outpatient oncology clinic.

- Physicians. Andrew's family can expect to meet their primary oncologist who will see them as outpatients. They will also meet oncology fellows and residents at various points along their treatment.

- Nurse practitioner. These advanced practice nurses collaborate with the medical team to provide and coordinate the ongoing care of Andrew.

- A social worker. Social work professionals assigned to the pediatric oncology department assist Andrew's family through the financial and social issues of having a seriously ill child, and are members of the psychology team. Some centers have specific psychological support programs while others have psychologists and psychiatrists available on an as-needed basis.

- Members of the child life team. These individuals, with training in child development or having an earned degree in child life therapy, are introduced to Andrew and his family early into their treatment and continue to care for Andrew's therapeutic play needs throughout the hospital stay and on return hospitalizations. Members of the child life team vary from center to center, but can include child life specialists, art and music therapists, hospitalbased schoolteachers and recreational therapists.

This next case study describes a 25-year-old female, Christy, who is long-term survivor of Hodgkin's disease. Christy was diagnosed at age 16 years when she presented with cervical lymphadenopathy and on work-up was found to have mediastinal involvement. Her treatment included Adriamycin $200 \mathrm{mg} / \mathrm{m}^{2}$, Bleomycin 80 units $/ \mathrm{m}^{2}$, Vinblastine, Dacarbazine, and $2100 \mathrm{cGy}$ radiation to the neck and mantle fields. She is followed in a survivorship program on a yearly basis. Christy is at risk for late cardiac complications, including diminished left ventricular function, pericarditis, valvular disease, early coronary artery disease, and arrhythmia. She requires yearly follow-up for cardiac signs/symptoms and every 2 years has echocardiogram (EKG) and lipid profile/fasting glucose. Patient education includes diet and exercise to reduce risk of cardiac complications. Her radiation and Bleomycin put her at risk for late pulmonary compromise including fibrosis, interstitial pneumonitis, and restrictive/obstructive lung disease. She is evaluated yearly for pulmonary symptoms and has had a baseline pulmonary function test including diffusing capacity of the lung for carbon monoxide (DLCO) and spirometry. She receives counseling on good pulmonary hygiene (eg, smoking cessation) and appropriate vaccinations (Influenza and Pneumoccal), which she obtains at her primary carer.

Christy is also at risk for a second malignancy, most notably secondary breast cancer due to her chest irradiation. She was instructed on self-breast exam upon entry to the survivorship program, and at 25 she began monitoring yearly with mammogram and breast magnetic resonance imaging (MRI). During her first exam, she was noted to have a density in the left breast and was referred for further radiologic evaluation with ultrasound. The density was 
ultimately biopsied and pathology confirmed benign fibrotic changes. She is now followed in a high-risk breast program with increased screening and exams. Christy's thyroid was in the radiation field, which puts her at risk for thyroid dysfunction. She is monitored yearly with physical exam and thyroid function tests; 2 years ago, she was noted to have an elevated thyroid-stimulating hormone (TSH) and started on thyroid supplementation to decrease her risk of developing nodules or malignancy. In addition, a social worker evaluates Christy yearly for any psychosocial sequelae of therapy.

\section{Challenges and future directions}

A major challenge facing childhood cancer and multidisciplinary care is the cost of providing care and financing scientific advances in an era of diminishing health care funds. It is estimated to cost US\$509 000 (1998) annually to treat a child with cancer; this cost includes physician, hospital, outpatient, and laboratory services. ${ }^{68}$ There were no studies identified that assess the cost effectiveness of multidisciplinary care but program evaluations that assess financial outcomes may become necessary to justify this approach.

The National Cancer Institute (NCI) is the primary funding agency for childhood cancer research in the USA. The NCI budget for pediatric cancer research in the fiscal year 2009 was US\$192.8 million and includes multidisciplinary research in areas of diagnosis, treatment, biology, etiology, prevention, and outcomes research. ${ }^{69}$ Given the current economic climate, budget cuts threaten to diminish research funding and it is expected that there will be greater competition for research dollars. It may be necessary to incorporate health economists as part of the multidisciplinary team to insure resources are appropriately utilized to yield the greatest benefits.

\section{Conclusion}

This paper illustrates the importance of multidisciplinary care in pediatric oncology. There are few diseases as complex as childhood cancers, which have seen the proliferation of effective therapies over a matter of several decades. The impressive advances in the field would not be possible without the collaboration and cooperation of professionals across the scope of healthcare. Because of these advances, families who have to face the diagnosis of childhood cancer now have reason to be hopeful that their child will survive and go on to have a good QOL.

\section{Disclosure}

The authors declare no conflicts of interest in relation to this paper.

\section{References}

1. Hewitt M, Weiner SL, Simone JV, editors. Childhood Cancer Survivorship: Improving Care and Quality. Washington, DC: The National Academy Press; 2003.

2. Wolff JA. History of pediatric oncology. Pediatr Hematol Oncol. 1991;8:88-91.

3. Farber S. Chemotherapy in the treatment of leukemia and Wilms' tumor. JAMA. 1966;198:826.

4. Wilbur JR. Combination chemotherapy for embryonal rhabdomyosarcoma. Cancer Chemother Rep. 1974;58(2):281-284.

5. Meadows AT. Pediatric cancer survivors: past history and future challenges. Available at: http://www.iom.edu/ /media/Files/Activity\%20 Files/Disease/NCPF/BackgroundPaperPediatricCancerSurvivors PastHistoryandFutureChallanges.pdf. Accessed April 28, 2011.

6. Djerassi I, Farber S, Abir E, Neikirk W. Continuous infusion of methotrexate in children with acute leukemia. Cancer. 1967;20: 233-242.

7. Aur RJA, Simone J, Hustu HO, et al. Central nervous system therapy and combination chemotherapy of childhood lymphocytic leukemia. Blood. 1971;37(3):272-281.

8. Aur RJA, Simone JV, Verzosa MS, Hustu HO, Pinkel DP, Barker LF. Leukoencephalopathy in children with acute lymphocytic leukemia receiving preventive central nervous system therapy [author's translation]. Sangre (Barc). 1978;23:1-12.

9. Rubin CM, Le Beau MM, Mick R, et al. Impact of chromosomal translocations on prognosis in childhood acute lymphoblastic leukemia. J Clin Oncol. 1991;9:2183-2192.

10. Borella L, Sen L. T cell surface markers on lymphoblasts from acute lymphocytic leukemia. J Immunol. 1973;111:1257-1260.

11. Dow LW, Borella L, Sen L, et al. Initial prognostic factors and lymphoblast-erythrocyte rosette formation in 109 children with acute lymphoblastic leukemia. Blood. 1977;50:671-682.

12. Crist WM, Pui CH. Clinical implications of cytogenetic and molecular analyses of pediatric acute lymphoblastic leukemia. Stem Cells. 1993; $11: 81-87$.

13. Jeha S, Pui CH. Risk-adapted treatment of pediatric acute lymphoblastic leukemia. Hematol Oncol Clin North Am. 2009;23:973-990.

14. Federman N, Bernthal N, Eilber FC, Tap WD. The multidisciplinary management of osteosarcoma. Curr Treat Options Oncol. 2009;10: 82-93.

15. Abed R, Grimer R. Surgical modalities in the treatment of bone sarcoma in children. Cancer Treat Rev. 2010;36:342-347.

16. Rao BN, Tsuchida Y, Kaneko M, Spicer RD, Plaschkes J. The surgeon and the child with cancer: a report of the International Society of Pediatric Surgical Oncology (IPSO). Med Pediatr Oncol. 2000;34: 424-428.

17. American Academy of Pediatrics. Section on hematology/oncology. Policy statement on guidelines for pediatric cancer centers. Pediatrics. 2004;113(6):1833-1835.

18. American Academy of Pediatrics section statement: Guidelines for the pediatric cancer center and role of such centers in diagnosis and treatment. Pediatrics. 2004;113:1833-1835.

19. Kellie SJ, Howard SC. Global child health priorities: what role for paediatric oncologists? Eur J Cancer. 2008;44:2388-2396.

20. Howard SC, Pedrosa M, Lins M, et al. Establishment of a pediatric oncology program and outcomes of childhood acute lymphoblastic leukemia in a resource-poor area. JAMA. 2004;291:2471-2475.

21. Raab CP, Gartner JC. Diagnosis of childhood cancer. Prim Care. 2009; $36: 671-684$ 
22. Carlson CA, Hobbie WL, Brogna M, Ginsberg JP. A multidisciplinary model of care for childhood cancer survivors with complex medical needs. J Pediatr Oncol Nurs. 2008;25:7-13.

23. Kurt BA, Armstrong GT, Cash DK, et al. Primary care management of the childhoood cancer survivor. J Pediatr. 2008;152:458-466.

24. Kline NE. Multidisciplinary care of the pediatric oncology patient. J Pediatr Oncol Nurs. 2005;22:193.

25. Tracey E, DiTaranto S, Womer R. Evolution of a rapid hydration protocol. J Pediatr Oncol Nurs. 2004;21:22-26.

26. Ruccione K, Hinds PS, Wallace JD, Kelly KP; Children's Oncology Group Nursing Discipline. Creating a novel structure for nursing research in a cooperative clinical trials group: The Children's Oncology Group experience. Semin Oncol Nurs. 2005;21(2):79-88.

27. Ruccione K, Kelly KP. State of the science: pediatric oncology nursing in cooperative clinical trials groups comes of age. Sem Oncol Nurs. 2000; $16: 253-260$

28. Hinds PS, Hockenberry MJ, Gattuso JS, et al. Dexamethasone alters sleep and fatigue in pediatric patients with acute lymphoblastic leukemia. Cancer. 2007;15:2321-2330.

29. Reaman GH. Pediatric cancer research from past successes through collaboration to future transdisciplinary research. J Pediatr Oncol Nurs. 2004;21:123-127.

30. Ruccione K. The legacy of pediatric oncology nursing in advancing survivorship research and clinical care. J Pediatr Oncol Nurs. 2009;26: 255-265.

31. Pek JH, Chan YH, Yeoh AE, Quah TC, Tan PL, Aung L. Health-related quality of life in children with cancer undergoing treatment: a first look at the Singapore experience. Ann Acad Med Singapore. 2010;39: 43-48.

32. Eiser C. Beyond survival: quality of life and follow-up after childhood cancer. J Pediatr Psychol. 2007;32:1140-1150.

33. Reimers TS, Mortensen EL, Nysom K, Schmiegelow K. Health-related quality of life in long-term survivors of childhood brain tumors. Pediatr Blood Cancer. 2009;53:1086-1091.

34. Blaauwbroek R, Groenier KH, Kamps WA, Meyboom-de JB, Postma A. Late effects in adult survivors of childhood cancer: the need for life-long follow-up. Ann Oncol. 2007;18:1898-1902.

35. Meeske KA, Patel SK, Palmer SN, Nelson MB, Parow AM. Factors associated with health-related quality of life in pediatric cancer survivors. Pediatr Blood Cancer. 2007;49:298-305.

36. Reaman GH. Pediatric oncology: current views and outcomes. Pediatr Clin N Am. 2002;49:1305-1318.

37. Bertolone K. Pediatric oncology: past presnet, and new modlaities of treatment. J Intraven Nurs. 1997;20:136-140.

38. Ross JA, Olshan AF. Pediatric cancer in the United States: the Children's Oncology Group epidemiology reaserch program. Cancer Epidemiol Biomarkers Prev. 2004;13(10):1552-1554.

39. Basu SK, Fernandez ID, Fisher SG, Asselin BL, Lyman GH. Length of stay and mortality associated with febrile neutropenia among children with cancer. J Clin Oncol. 2005;23:7958-7966.

40. Pasqualotto AC, Denning DW. New and emerging treatments for fungal infections. J Antimicrob Chemother. 2008;61(Suppl 1):i19-i30.

41. Araya CE, Lew JF, Fennell RS III, Neiberger RE, Dharnidharka VR. Intermediate-dose cidofovir without probenecid in the treatment of BK virus allograft nephropathy. Pediatr Transplant. 2006;10:32-37.

42. Orudjev E, Lange BJ. Evolving concepts of management of febrile neutropenia in children with cancer. Med Pediatr Oncol. 2002;39: 77-85.

43. Santolaya ME. Supportive care in children. Curr Opin Oncol. 2010;22: 323-329.

44. Hawkins J. Supportive care: managing febrile neutropenia. Paediatr Nurs. 2009;21:33-37.

45. Barton SJ, Chase T, Latham B, Rayens MK. Comparing two methods to obtain blood specimens from pediatric central venous catheters. J Pediatr Oncol Nurs. 2004;21:320-326.
46. Horvath B, Norville R, Lee D, Hyde A, Gregurich M, Hockenberry M. Reducing central venous catheter-related bloodstream infections in children with cancer. Oncol Nurs Forum. 2009;36:232-238.

47. Pinkerton CR, Williams D, Wootton C, Meller ST, McElwain TJ. 5-HT3 antagonist ondansetron-an effective outpatient antiemetic in cancer treatment. Arch Dis Child. 1990;65:822-825.

48. Hedstrom M, Haglund K, Skolin I, von EL. Distressing events for children and adolescents with cancer: child, parent, and nurse perceptions. J Pediatr Oncol Nurs. 2003;20:120-132.

49. Williams PD, Schmideskamp J, Ridder EL, Williams AR. Symptom monitoring and dependent care during cancer treatment in children: pilot study. Cancer Nurs. 2006;29:188-197.

50. Dupuis LL, Taddio A, Kerr EN, Kelly A, MacKeigan L. Development and validation of the pediatric nausea assessment tool for use in children receiving antineoplastic agents. Pharmacotherapy. 2006;26: 1221-1231.

51. Linder LA. Measuring physical symptoms in children and adolescents with cancer. Cancer Nurs. 2005;28:16-26.

52. Phillips RS, Gopaul S, Gibson F, et al. Antiemetic medication for prevention and treatment of chemotherapy induced nausea and vomiting in childhood. Cochrane Database Syst Rev. 2010:CD007786.

53. McGrath PA. Development of the World Health Organization guidelines on cancer pain relief and palliative care in children. J Pain Symptom Manage. 1996;12:87-92.

54. Geeta MG, Geetha P, Ajithkumar VT, Krishnakumar P, Kumar KS, Mathews L. Management of pain in leukemic children using the WHO analgesic ladder. Indian J Pediatr. 2010;77:665-668.

55. Zernikow B, Smale H, Michel E, Hasan C, Jorch N, Andler W. Paediatric cancer pain management using the WHO analgesic ladder-results of a prospective analysis from 2265 treatment days during a quality improvement study. Eur J Pain. 2006;10:587-595.

56. Post-White J, Sencer S, Fitzgerald M. Complementary and alternative treatments in children with cancer. In: Kelly K, Baggott C, Foley G, editors. Nursing Care of the Child with Cancer. 2nd ed. Philadelphia: Saunders; 2003:256-263.

57. Post-White J. Complementary and alternative medicine in pediatric oncology. J Pediatr Oncol Nurs. 2006;23:244-253.

58. Post-White J, Hawks R. Complementary and alternative medicine in pediatric oncology. Sem Oncol Nurs. 2005;21:107-114

59. Wild MR, Espie CA. The efficacy of hypnosis in the reduction of procedural pain and distress in pediatric oncology: a systematic review. J Dev Behav Pediatr. 2004;25:207-213.

60. Bishop FL, Prescott P, Chan YK, Saville J, von Elm E, Lewith GT. Prevalence of complementary medicine use in pediatric cancer: a systematic review. Pediatrics. 2010;125:768-776.

61. Hawks R. Complementary and alternative medicine research initiatives in the Children's Oncology Group and the role of the pediatric oncology nurse. J Pediatr Oncol Nurs. 2006;23:261-264.

62. Post-White J, Hawks R, O’Mara A, Ott MJ. Future directions of CAM research in pediatric oncology. J Pediatr Oncol Nurs. 2006;23: 265-268.

63. Zebrack B, Zeltzer L. Quality of life issues and cancer survivorship. Curr Probl Cancer. 2003;27:198-211.

64. Children's Oncology Group. www.childrensoncologygroup.org/.

65. CureSearch. Children's cancer information. Available at: http://www. curesearch.org/. Accessed April 28, 2011.

66. Robison LL, Armstrong GT, Boice JD, et al. The Childhood Cancer Survivor Study: a National Cancer Institute-supported resource for outcome and intervention research. J Clin Oncol. 2009;27: 2308-2318.

67. CureSearch. Long-term follow-up guidelines for survivors of childhood, adolescent, and young adult cancers. Available at: http://www. childrensoncologygroup.org/disc/le/pdf/LTFUGuidelines.pdf. Accessed April 28, 2011. 
68. Landrigan PJ, Schechter CB, Lipton JM, Fahs MC, Schwartz J. Environmental pollutants and disease in American children: estimates of morbidity, mortality, and costs for lead poisoning, asthma, cancer, and developmental disabilities. Environ Health Perspect. 2002;110(7):721-728.
69. National Cancer Institute. A snapshot of pediatric cancers. Available at: http://www.cancer.gov/aboutnci/servingpeople/pediatric-snapshot. pdf. Accessed April 28, 2011.

\section{Publish your work in this journal}

The Journal of Multidisciplinary Healthcare is an international, peer-reviewed open-access journal that aims to represent and publish research in healthcare areas delivered by practitioners of different disciplines. This includes studies and reviews conducted by multidisciplinary teams as well as research which evaluates the results or conduct of such teams or healthcare processes in general. The journal covers a wide range of areas and welcomes submission from practitioners at all levels, from all over the world. The manuscript management system is completely online and includes a very quick and fair peer-review system. Visit http://www.dovepress.com/testimonials.php to read real quotes from published authors.

Submit your manuscript here: http://www.dovepress.com/journal-of-multidisciplinary-healthcare-journal 